\title{
Rotational alignment of the tibial component in total knee arthroplasty: the anterior tibial cortex is a reliable landmark
}

\author{
ANDREA BALDINI', PIER FRANCESCO INDELLI ${ }^{2}$, LAPO DE LUCA ${ }^{3}$, PIERPAOLO CERULLI \\ MARIANI $^{4}$, MASSIMILIANO MARCUCCI ${ }^{2}$ \\ ${ }^{1}$ Humanitas Institute, Milan, Italy \\ ${ }^{2}$ Orthopaedic Clinic, University of Florence, CESAT, Fucecchio, Italy and The Breyer Center for Overseas Studies, \\ Stanford University, Florence, Italy \\ ${ }^{3}$ CTO, Florence, Italy \\ ${ }^{4}$ Misericordia and Dolce Hospital, Prato, Italy
}

\begin{abstract}
Purpose: to compare the anterior tibial surface curvature, the Akagi's line and the medial third of the tibial tubercle in order to assess which is the most reliable landmark for correct tibial component rotational positioning in total knee arthroplasty.

Methods: three independent investigators reviewed 124 knee MRI scans. The most suitable tibial baseplate tracing for the Nexgen Total Knee System (Zimmer, Warsaw, USA) was superimposed on the scan matching the anterior tibial cortex with the anterior aspect of the baseplate. The rotation of the tibial baseplate tracing was calculated with respect to the transepicondylar axis (TEA), the medial third of the tibial tubercle line, Akagi's line and the femoral posterior condylar axis (PCA). Customized software was created and used for analysis of the MRI datasets. The reliability of each measurement was then calculated by using the intraclass correlation coefficient for interobserver agreement.

Results: observer agreement on the position of the Akagi's line was within $3^{\circ}$ in $64 \%$ of the cases and within $5^{\circ}$ in $85 \%$ of the cases. Agreement on the position of the medial third of the tibial tubercle was
\end{abstract}

\section{Corresponding Author:}

Pier Francesco Indelli, MD

Orthopaedic Clinic, University of Florence,

CESAT Fucecchio

Piazza Lavagnini 1, 50054 Fucecchio (FI), Italy

E-mail: pierfrancesco.indelli@unifi.it within $3^{\circ}$ in $29 \%$ of the cases and within $5^{\circ}$ in $70 \%$ of the cases. Agreement on the localization of the anterior tibial surface curvature was within $3^{\circ}$ in $89 \%$ of the cases and within $5^{\circ}$ in $99 \%$ of the cases. Component alignment along the anterior cortex guaranteed full matching $\pm 3^{\circ}$ with the epicondylar axis in $75 \%$ of the knees.

Conclusions: the anterior tibial surface curvature was found to be a more reliable and more easily identifiable landmark for correct tibial component alignment than either Akagi's line or the medial third of the tibialtubercle.

Level of evidence: level III, retrospective cohort study.

Key Words: arthroplasty, alignment, bone landmarks, knee, magnetic resonance, TKA.

\section{Introduction}

Restoration of the mechanical axis and soft tissue balancing are well-established key factors for a successful total knee arthroplasty (TKA), an operation that continues to have a considerable failure rate. Many studies have reported a poor functional outcome following rotational malalignment of the femoral and tibial components (1-3). Rotational malalignment may lead to patellar maltracking, anterior knee pain, flexion instability and premature wear of the polyethylene inlay. The rotational alignment of the femoral component has been extensively studied and useful reference axes for setting proper femoral rotation have been esta- 
blished, including the posterior condylar axis (PCA), the midtrochlear line (Whiteside's line) and the transepicondylar axis (TEA)(4-6). As a result, many femoral cutting guides use the transepicondylar line as a reference for rotational alignment of the femoral component.

Less attention has been paid to rotational alignment of the tibial component, for which there is still no established standard reference in the current literature. Currently, two techniques are used to determine tibial rotation in TKA. The first utilizes anatomical landmarks, while the second is a range-of-motion (ROM) technique. Historically, the anatomical landmark technique used conventional references, both extra-articular (i.e. the transmalleolaraxis, the second metatarsal axis and the tibial tubercle) and intra-articular (i.e. the posterior tibial condylar line, the transcondylar tibial line, and the line between the tibial spines). More recently, various sagittal planes have been described including a line perpendicular to the posterior joint surface passing through the medial third of the tibial tubercle (7) and a line passing through the middle of the posterior cruciate ligament perpendicularly to the projected femoral TEA (Akagi's line) (8).

Unfortunately, many of these references vary among patients, are difficult to establish, and are therefore unreliable. The ROM technique aligns the tibial component according to the rotational alignment of the femoral component during trial reduction by means of a "self-seeking method". Unfortunately, this method induces the risk of transferring a femoral malrotation to the tibia (1). None of these methods has been universally adopted.

The aim of this study was to define an easily identifiable landmark and to propose a reliable method for ideal positioning of the tibial component in TKA. We set out to ascertain whether there is an optimal way of orienting the tibial component in TKA, starting from the assumption that a single area would be a better and more readily identifiable landmark than a single point or a line, as previously described (6-8). Our hypothesis was that the anterior tibial surface curvature is a more reliable landmark for correct tibial component rotational positioning in TKA with respect to Akagi's line and the medial third of the tibial tubercle.

\section{Methods}

We analyzed 124 magnetic resonance imaging (MRI) knee scans from 124 patients (69 women and 55 men) with a mean age of 42 years (18 to 74 years). All scans were performed using an open-style MRI scanner (MAGNETOM® MRI system; Siemens, Munich, Germany), positioning the knee in full extension with the second metatarsal axis in a vertical position: all images had a thickness of $2 \mathrm{~mm}$ with $3 \mathrm{~mm}$ in reconstructive increments from the distal metaphysis to the tibial tubercle. All scans were performed in patients with suspected ligament or cartilage damage. None of the knees showed the presence of osteoarthritis, ligament lesions, significant meniscal lesions, or flexion contracture.

The femoral TEA and the femoral PCA were identified on a single axial scan of the distal femur (Fig. 1), while the projection of the medial third of the tibial tubercle on the anterior tibial cortex was identified on an axial MRI scan of the proximal tibia (Fig. 2). For each knee, the TEA, PCA and medial third of the tibial tubercle were identified on axial MRI scan of the proximal tibia. The geometric center of the tibial plateau was also identified (Fig. 3). A line was first drawn from the center of the tibial plateau to the medial third of the tubercle (A), after which the line perpendicular to the TEA passing through the center of the tibial

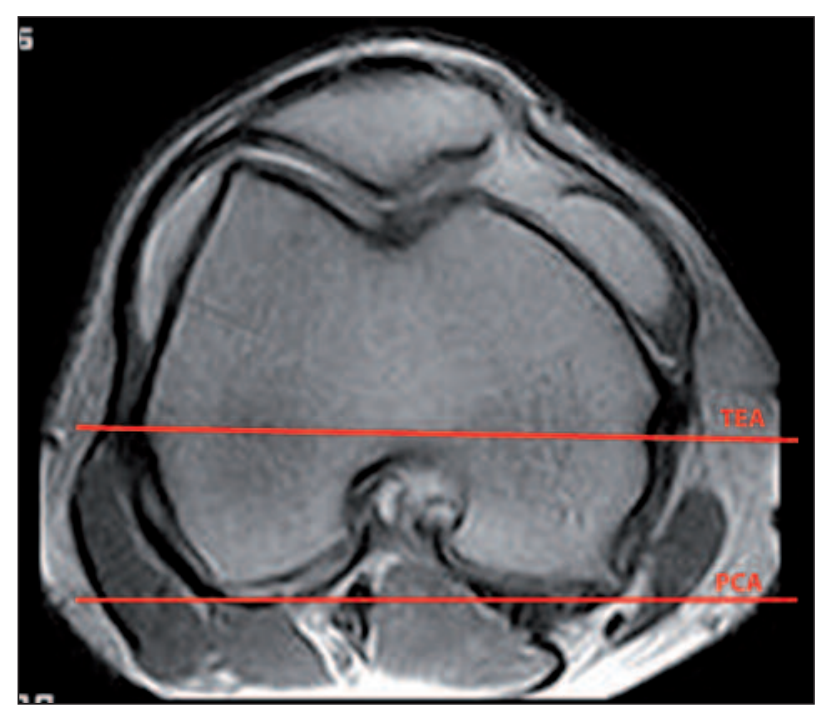

Fig. 1. Axial MRI scan of the distal femur: transepicondylar axis (TEA) and femoral posterior condylar axis (PCA). 


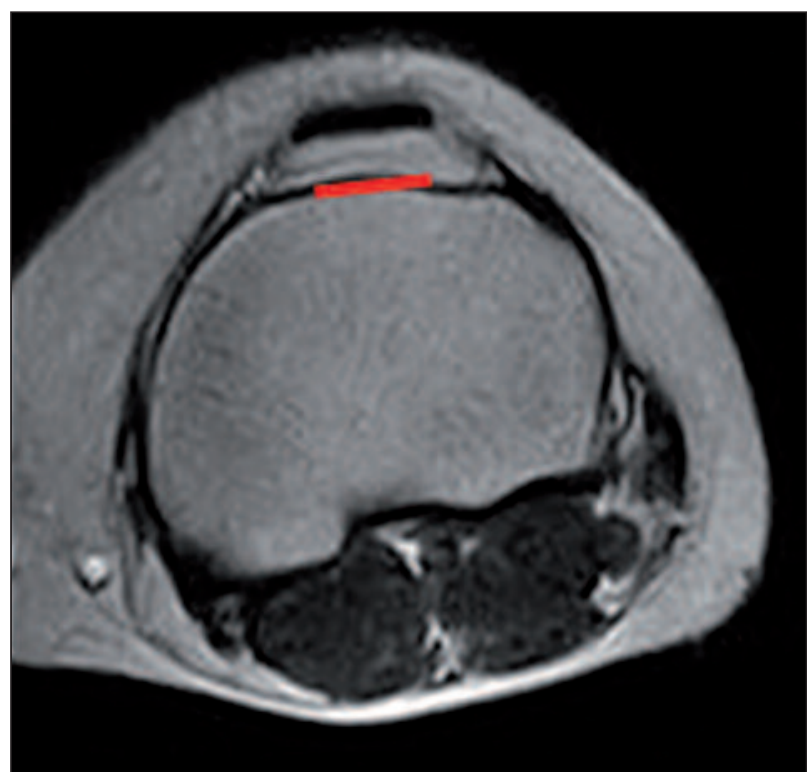

Fig. 2. Axial MRI scan of the proximal tibia: projection of the medial third of the tibial tubercle on the anterior tibial cortex.

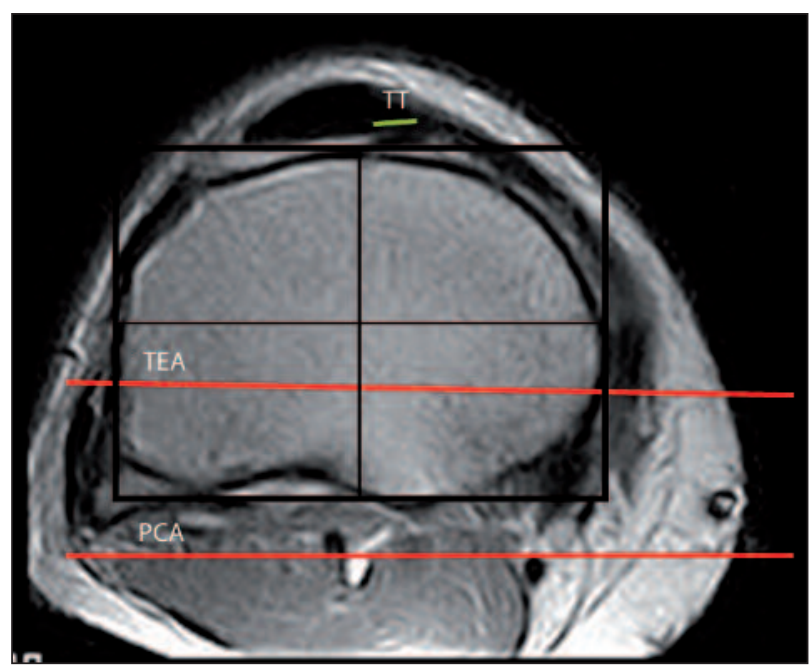

Fig. 3. Axial MRI scan of the proximal tibia: identification of the geometric center of the tibialplateau. TT = projection of the medial third of the tibial tubercle on the anterior tibial cortex; TEA = transepicondylar axis; PCA = femoral posterior condylar axis.

plateau (Fig. 4) was identified (B). At this point, Akagi's line was drawn (Fig. 5). This landmark is defined by a line starting at the medial third of the tibial tubercle and ending at the center of the tibial insertion of the posterior cruciate ligament. The most suitable tibial baseplate tracing (size 3 to 8 ) for the Nexgen

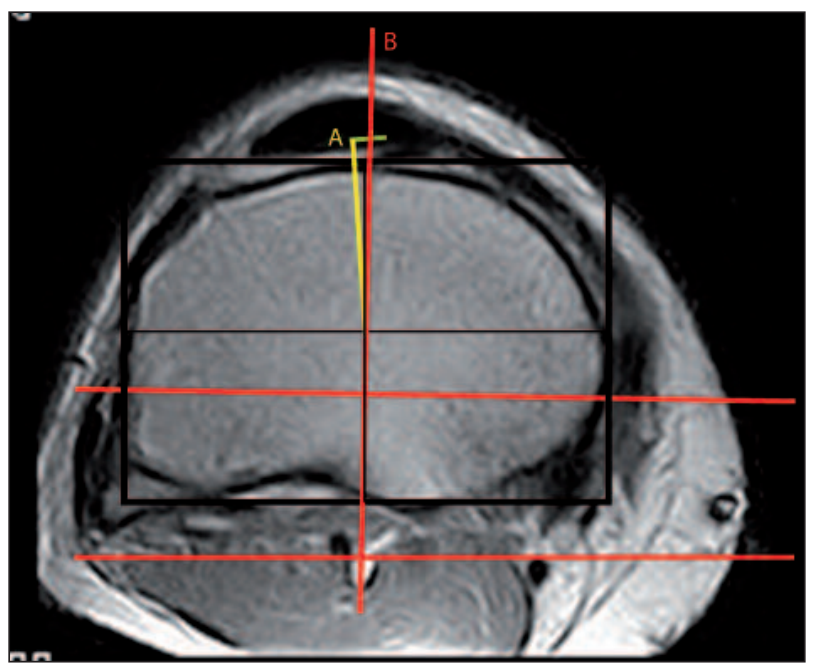

Fig. 4. Axial MRI scan of the proximal tibia: A) Line A: conjunction between the geometric center of the tibial plateau and the projection of the medial third of the tibial tubercle on the anterior tibial cortex; B) Line B: perpendicular conjunction between the geometric center of the tibial plateau and the projection of the TEA.

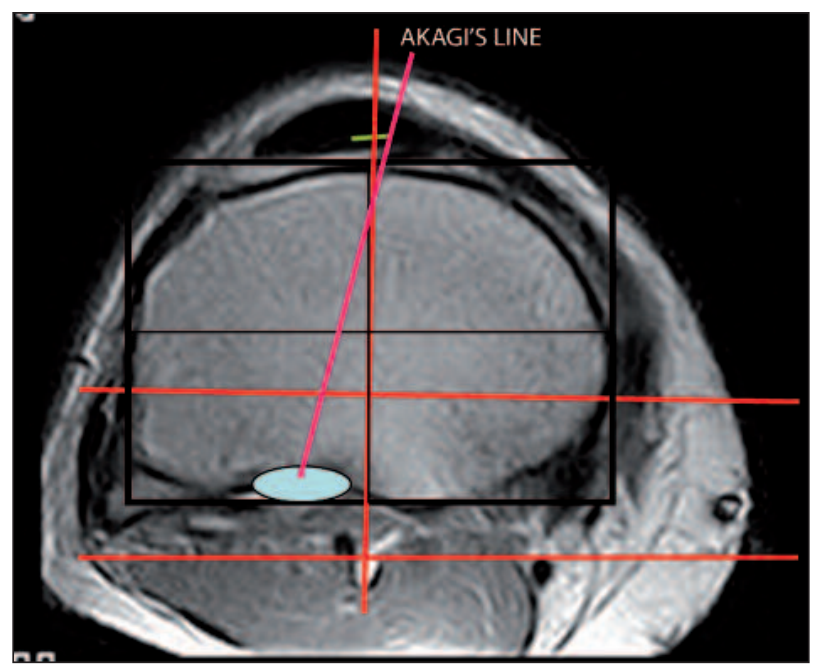

Fig. 5. Axial MRI scan of the proximal tibia: Akagi's line. This landmark is defined by a line starting at the medial third of the tibial tubercle and ending at the center of the tibial insertion of the posterior cruciate ligament.

Total Knee System (Zimmer, Warsaw, USA) was superimposed on the scan, matching the anterior tibial cortex with the anterior surface of the baseplate (Fig. 6).The rotation of the tibial baseplate tracing was calculated with respect to the TEA, medial third of the tibial tubercle line, Akagi's line and PCA. 


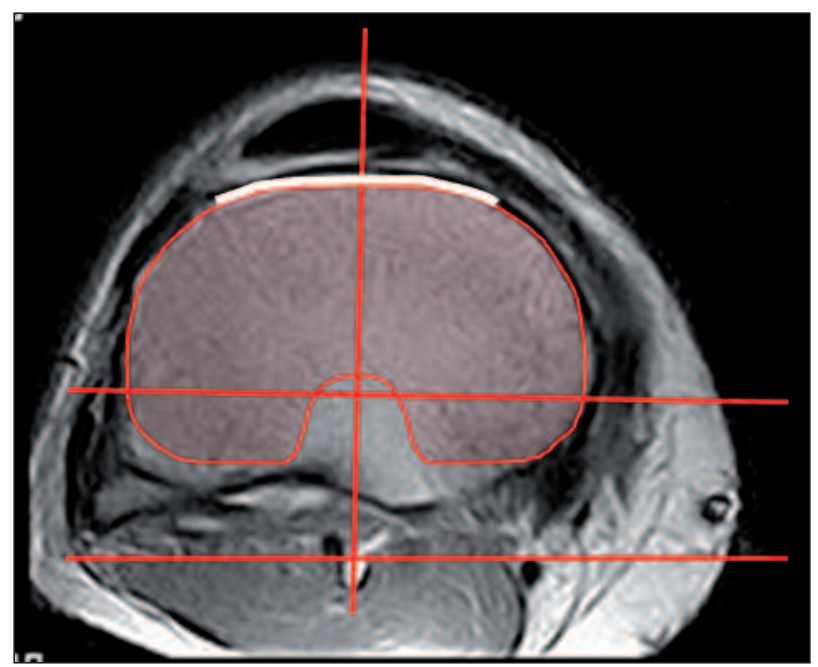

Fig. 6. Tibial baseplate tracing (Nexgen Total Knee System, Zimmer, Warsaw, USA) superimposed on the MRI matching the anterior tibial cortex with the anterior surface of the baseplate. The posteromedial corner is not covered: this is an indirect sign of sufficient external rotation of the tibial component.

Customized software was created and used for analysis of the MRI datasets. All axial images were evaluated independently by three observers (PFI, PCM, AB). They independently repeated the entire process, from point gathering to measurement of angles. The reliability of each measurement was then calculated by using the intraclass correlation coefficient for interobserver agreement. The coefficient value is reported as an average of multiple pairwise comparisons (PFI vs PCM; PFI vs $\mathrm{AB}$; $\mathrm{PCM}$ vs $\mathrm{AB})$.

\section{Results}

\section{Akagi's line}

With regards to the position of Akagi's line, the three observers showed agreement within $3^{\circ}$ in $64 \%$ of the cases and within $5^{\circ}$ (minimum: $16^{\circ}$, maximum: $7^{\circ}$ ) in $85 \%$ of the cases. The average intraclass correlation coefficient was 0.923 (PFI vs PCM: 0.910; PFI vs AB: 0.933; $\mathrm{PCM}$ vs $\mathrm{AB}$; 0.927). Use of this landmark might lead to internal rotation of the tibial component.

\section{Medial third of the tibial tubercle}

With regards to the position of the medial third of the tibial tubercle the three observers showed agreement within $3^{\circ}$ in $29 \%$ of the cases and within $5^{\circ}$ (minimum $-4^{\circ}$, maximum $+4^{\circ}$ ) in $70 \%$ of the cases. The average intraclass correlation coefficient was 0.881 (PFI vs PCM: 0.871; PFI vs AB: 0,897; PCM vs AB: 0.876 ). Use of this landmark led to an average $4.7^{\circ}$ ( \pm $\left.3.6^{\circ}\right)$ of external rotation of the tibial component with respect to the TEA.

\section{Anterior tibial surface curvature}

The three observers showed agreement on the localization of the anterior tibial surface curvature within $3^{\circ}$ in $89 \%$ of the cases and within $5^{\circ}$ (minimum $-1^{\circ}$, maximum $+4^{\circ}$ ) in $99 \%$ of the cases. The average intraclass correlation coefficient was 0.949 (PFI vsPCM: 0.940; PFI vsAB: 0.961 ; PCM vsAB: 0.947$)$. In $75 \%$ of the cases, component alignment along the anterior cortex guaranteed full matching $\left( \pm 3^{\circ}\right)$ with the epicondylar axis in extension, with minor errors in external rotation.

\section{Discussion}

Rotational malalignment has been shown to be a major cause of medium-term failure in TKA (1$3)$.While the TEA is universally recognized as a primary reference for femoral rotational alignment (9$11)$, there is no consensus as regards tibial rotational alignment. In fact, many different anatomical landmarks have previously been suggested as the best references for tibial component rotational alignment in TKA (4-6, 8).

Many surgeons prefer a single point as a reference. Incavo et al. (12) suggested aligning the mid-axis line of the tibial tray with a point close to the medial third of the patellar tendon. Lützner et al. (13) showed better femorotibial rotational alignment when using the medial third of the tibial tubercle as a landmark. Barrack et al. (14) suggested using the most prominent point of the tibial tubercle for correct tibial component alignment. Unfortunately, Cobb et al. (15), in a cadaveric study, found a very large variation in the position of the center of the tibial tubercle between the knees studied. Ikeuchi et al. (6) indicated the medial border of the attachment of the patellar tendon as the best landmark for tibial rotational positioning. 
Recently, Rossi et al.(16), in a cadaveric study, validated the posterolateral tibial corner as a reliable reference point: the identification of this point requires complete exposure of the tibial plateau, which is difficult to obtain in many knees.

Other studies have suggested the use of an axis or a sagittal plane in place of a single-point landmark for correct rotational alignment. Akagi et al. (8) described a line perpendicular to the projected femoral TEA, starting at the medial third of the tibial tubercle and directed towards the middle of the tibial insertion of the posterior cruciate ligament. Dalury (17) proposed using a line starting from the mid-point between the tibial spines and passing $1 \mathrm{~mm}$ medially to the medial border of the tibial tubercle. Luo (18) proposed using a line lying perpendicular to the posterior joint surface and passing through the medial third of the tibial tubercle. Unfortunately, many sagittal axes are not easily and reliably identifiable during surgery. Graw et al. (19) showed high variability of several sagittal axes in relation to different tibial resection levels. Nagamine et al. (20) demonstrated that a sagittal anteroposterior axis was less reliable than the PCA for use in alignment in TKA.

Some surgeons, including Incavo et al. (21) and Westrich et al. (22), prefer the use of an asymmetrical component, maximizing tibial cover in order to provide the best stability and load transfer in TKA. In our experience, tibial cover itself is not sufficient to guarantee a satisfactory tibial rotational alignment. Pagnano et al. (23) demonstrated that rotational malalignment in TKA is not correctable by the use of a mobile-bearing option.

Siston et al. (24) affirmed that neither the axis technique nor the single-point reference technique establish a correct tibial rotational alignment, and suggested, instead, the use of computer-assisted techniques for correct rotational alignment in TKA.

Eckhoff et al. (25) suggested using the ROM technique instead of anatomical landmarks for correct component alignment in TKA. They put the knee through a full range of flexion and extension, allowing the tibial tray to find its own best position in relation to the femoral component. The ROM technique is highly dependent on the rotational orientation of the femoral component and the soft tissue balancing; many authors $(6,26,27)$ did not suggest this technique because of the risk of positioning the tibial component with excessive internal rotation.

The present study showed that the anterior tibial cortex is a reliable and easily identifiable landmark for correct tibial component positioning. It allows satisfactory parallelism between the mediolateral axis of the tibial component and the epicondylar axis. This technique also makes it possible to determine the correct rotational alignment of the prosthetic components with respect to the extensor mechanism, avoiding many complications related to the patellofemoral joint (28). The occurrence of patellofemoral complications after TKA is still the most frequently reported cause of pain and the most frequently reported reason for revision surgery (2).

Our study has several limitations. It failed to establish whether there is an overall optimal orientation of the tibial component during TKA. However, it provides a reproducible method for correct rotational alignment of the tibial component. In this study, we used a symmetrical tibial baseplate tracing. It is possible that the use of an anteriorly asymmetrical tracing might lead to excessive internal rotation of the component, if the "curve-on-curve" technique is chosen intraoperatively. The use of an MRI scan as a preoperative planning tool may be questionable. However, we believe that MRIbased preoperative measurements overcome intraoperative limitations while accounting for the individual anatomy of each patient, thus helping to optimize component rotation. Finally, the results of our study may not apply to the severely deformed knee. We purposely studied knees without any major malalignment. We hypothesize that the use of tibial component alignment following an anterior tibial surface without major osteoarthritic deformity might be appropriate for deformed knees too. Patel et al. (29) showed that the degree of preoperative osteoarthritic deformity did not influence the use of the TEA as a reliable rotational landmark in TKA. Rotational malalignment of components may cause chronic pain or early failure in TKA. Our study showed that the anterior tibial cortex is an easily identifiable and reliable landmark for correct rotational alignment of the tibial component when compared with Akagi's line and the medial third of the tibial tubercle. In fact, tibial component alignment along the anterior 
cortex guaranteed full matching $\pm 3^{\circ}$ with the TEA in $75 \%$ of the cases, with minor errors in external rotation. In conclusion, the anterior tibial surface curvature was found to be a more reliable and more easily identifiable landmark for correct tibial component alignment than either Akagi's line or the medial third of the tibial tubercle.

\section{References}

1. Benjamin J. Component alignment in total knee arthroplasty. Instr Course Lect 2006;55:405-412.

2. Patel J, Ries MD, Bozic KJ. Extensor mechanism complications after total knee arthroplasty. Instr Course Lect 2008;57:283-294.

3. Windsor RE, Scuderi GR, Moran MC, et al.Mechanisms of failure of the femoral and tibial components in total knee arthroplasty. Clin Orthop Relat Res 1989; 248:15-19.

4. Aglietti P, Sensi L, Cuomo P, et al. Rotational position of femoral and tibial components in TKA using the femoral transepicondylar axis. Clin Orthop Relat Res 2008; 466:2751-2755

5. Chowdhury EA, Porter ML. How is the tibial tray aligned to the femoral prosthesis in a total knee arthroplasty? A survey of opinion from BASK? Knee 2005; 12:79-80.

6. Ikeuchi M, Yamanaka N, Okanoue Y, et al. Determining the rotational alignment of tibialcomponent at total knee replacement: a comparison of two techniques. J Bone Joint Surg Br 2007; 89:45-49.

7. Uehara K, Kadoya Y, Kobayashi A, et al. Bone anatomy and rotational alignment in total knee arthroplasty. Clin Orthop Relat Res 2002; 402:196-201.

8. Akagi M, Oh M, Nonaka T, et al. An anteroposterior axis of the tibia for total knee arthroplasty. Clin Orthop Relat Res 2004; 420:213-219.

9. Akagi M, Mori S, Nishimura S, et al. Variability of extra articulartibial rotation references for total knee arthroplasty. Clin Orthop Relat Res 2005; 436:172-176.

10. Asano T, Akagi M, Nakamura T. The functional flexionextension axis of the knee corresponds to the surgical epicondylar axis: in vivo analysis using a biplanar image-matching technique. J Arthroplasty 2005; 20:1060-1067.

11. Olcott CW, Scott RD. A comparison of 4 intraoperative methods to determine femoral component rotation during total knee arthroplasty. J Arthroplasty 2000; 15:22-26.

12. Incavo SJ, Coughlin KM, Pappas C, et al. Anatomic rotational relationships of the proximal tibia, distal femur, and patella: implications for rotational alignment in total knee arthroplasty. J Arthroplasty 2003; 18:643-648.
13. Lützner J, Krummenauer F, Günther KP, et al. Rotational alignment of the tibial component in total knee arthroplasty is better at the medial third of tibial tuberosity than at the medial border.BMC Musculoskelet Disord 2010; 11:57.

14. Barrack RL, Schrader T, Bertot AJ, et al. Component rotation and anterior knee pain after total knee arthroplasty. Clin Orthop Relat Res 2001; 392:46-55.

15. Cobb JP, Dixon H, Dandachli W, et al. The anatomical tibial axis: reliable rotational orientation in knee replacement. J Bone Joint Surg Br 2008; 90:1032-1038.

16. Rossi R, Bruzzone M, Bonasia DE, et al. Evaluation of tibial rotational alignment in total knee arthroplasty: a cadaver study. Knee Surg Spots Traumatol Arthrosc 2010; 18:889893.

17. Dalury DF. Observations of the proximal tibia in total knee arthroplasty. Clin Orthop Relat Res 2001; 389:150-155.

18. Luo CF. Reference axes for reconstruction of the knee. Knee 2004; 11:251-257.

19. Graw BP, Harris AH, Tripuraneni KR, et al. Rotational references for total knee arthroplastytibial components change with level of resection. Clin Orthop Relat Res 2010; 468:2734-2738.

20. Nagamine R, Miura H, Inoue $Y$, et al. Reliability of the anteroposterior axis and the posterior condylar axis for determining rotational alignment of the femoral component in total knee arthroplasty. J Orthop Sci 1998; 3:194-198.

21. Incavo SJ, Ronchetti PJ, Howe JG, et al. Tibial plateau coverage in total knee arthroplasty. Clin Orthop Relat Res 1994; 299:81-85.

22. Westrich GH, Laskin RS, Haas SB, et al. Resection specimen analysis of tibial coverage in total knee arthroplasty. Clin Orthop Relat Res 1994; 309:163-175.

23. Pagnano MV, Trousdale RT, Stuart MJ, et al. Rotating platform knees did not improve patellar tracking: a prospective, randomized study of 240 primary total knee arthroplasties. Clin Orthop Relat Res 2004; 428:221-227.

24. Siston RA, Goodman SB, Patel JJ, et al. The high variability of tibial rotational alignment in total knee arthroplasty. Clin Orthop Relat Res 2006; 452:65-69.

25. Eckhoff DG, Metzger RG, Vandewalle MV. Malrotation associated with implant alignment technique in total knee arthroplasty. Clin Orthop Rel Res 1995; 321:28-31.

26. Romero J, Stähelin T, Wyss T, et al. Significance of axial rotation alignment of components of knee prostheses. Orthopade 2003; 32:461-468.

27. Hanada H, Whiteside LA, Steiger J, et al. Bone landmarks are more reliable than the tensioned gaps in TKA component alignment. Clin Orthop Relat Res 2007; 462:137-142.

28. Indelli PF, Marcucci M, Cariello D, et al. Contemporary femoral designs in total knee arthroplasty: effects on the patello-femoral congruence. Int Orthop 2012; 36:11671173.

29. Patel A, Yaffe M, McCoy B, et al. Should preoperative deformity determine femoral component rotation in TKA? J Bone Joint Surg Br 2012; 94 (Suppl XL):139. 